\title{
RISK MEASUREMENT WITH THE EQUIVALENT UTILITY PRINCIPLES
}

\author{
MICHEL DENUIT ${ }^{\star}$, JAN DHAENE ${ }^{\dagger, *}$, \\ MARC GOOVAERTS ${ }^{\dagger, \ddagger}$, ROB KAAS $^{\ddagger}$ \& ROGER LAEVEN R $^{\ddagger}$ \\ ${ }^{\star}$ Institut des Sciences Actuarielles \& Institut de Statistique \\ Université Catholique de Louvain \\ Louvain-la-Neuve, Belgium \\ ${ }^{\dagger}$ Catholic University of Leuven \\ Center for Risk and Insurance Studies \\ B-3000 Leuven, Belgium \\ $¥$ University of Amsterdam \\ Dept. of Quantitative Economics \\ 1018 WB Amsterdam, The Netherlands
}

December 20, 2005 


\begin{abstract}
Risk measures have been studied for several decades in the actuarial literature, where they appeared under the guise of premium calculation principles. Risk measures and properties that risk measures should satisfy have recently received considerable attention in the financial mathematics literature. Mathematically, a risk measure is a mapping from a class of random variables defined on some measurable space to the (extended) real line. Economically, a risk measure should capture the preferences of the decision-maker.

In incomplete financial markets, prices are no more unique but depend on the agents' attitudes towards risk. This paper complements the study initiated in DENUIT, DHAEne \& VAN WOUWE (1999) and considers several theories for decision under uncertainty: the classical expected utility paradigm, Yaari's dual approach, maximin expected utility theory, Choquet expected utility theory and Quiggin rank-dependent utility theory. Building on the actuarial equivalent utility pricing principle, broad classes of risk measures are generated, of which most classical risk measures appear to be particular cases. This approach shows that most risk measures studied recently in the financial literature disregard the utility concept (i.e. correspond to linear utilities), causing some deficiencies. Some alternatives proposed in the literature are discussed, based on exponential utilities.
\end{abstract}

Key words and phrases: Utility theories, risk measures, coherence, exponential utility, comonotonicity.

JEL-codes: D81, G10, G20. 


\section{Introduction and motivation}

\subsection{The need for risk measures}

In complete financial markets, hedging and arbitrage-free pricing are two sides of the same problem. According to the replication approach, the price of a contingent claim equals to price of the hedging portfolio. When the market is incomplete, however, there may be no replicating portfolio and the hedging strategy could involve a risky position. Moreover, there is an infinite number of martingale measures and each of them provides a different price for the contingent claim. This leads to a set of possible prices. Now, since the hedging of the contingent claim involves risk, the price of the hedging portfolio depends on the agent's attitude towards risk.

Note that the problem of market incompleteness is particularly relevant in insurance. This is due to the stochastic nature of insurance processes (with jumps), to the fact that many insurance risks are not actively traded in the (financial) market and to the fact that securitized insurance risks often have an underlying index rather than an underlying traded asset.

Recent years have witnessed the emergence in the financial literature of a sophisticated theory of risk measures. However, risk measures have been extensively studied in the actuarial literature for more than 30 years, in the guise of premium calculation principles; see, e.g., Bühlmann (1970) and Goovaerts, De ViJlder \& HaezenDONCK (1984). A risk measure is defined as a mapping from a set of random variables defined on a measurable space (representing the risks at hand) to the real numbers. We will often consider random variables as losses, or payments that need to be made. A negative outcome for the loss variable means that a gain has occurred. Then, $\varrho[X]$ denotes the real number that a general risk measure associates with the random variable $X$.

\subsection{How to define a risk measure?}

There are basically two ways to define a particular risk measure. In both cases, a set of axioms is given, but the axioms either describe how the risk is measured or describe how individuals make their decision under risk or uncertainty. Let us briefly explain these two approaches. First, a set of axioms for risk measurement can be given, that is regarded as reasonable in the situation under consideration. Then, the form of the risk measure is deduced from the agreed axioms. Risk measures are appropriate if, and only if, their characterizing axioms are. Axiomatizations can then be used to justify a risk measure but also to criticize it.

Second, a paradigm for decision under uncertainty can be selected, for instance the Von Neumann-Morgenstern expected utility theory, Yaari's dual utility theory or other generalized utility principles, for instance. Note that this selection also amounts to opting for a set of axioms, but this set explains how decision-makers choose between uncertain prospects; see, e.g., WAKKER (2004) for more details. The risk measure is then obtained by an equivalence principle: the decision-maker is indifferent between 
the cash-flow $\varrho[X]-X$ (corresponding to the case where $X$ is covered) and 0 (no coverage).

Both approaches for measuring risk will be considered in this paper. It will be seen that many classical risk measures can be obtained in both ways, which allows for a better understanding of their intrinsic properties. This will also enlighten their shortcomings in that most risk measures studied recently in the financial literature disregard utilities (considering only linear utility functions).

\subsection{The axiomatic approach to risk measurement}

Axioms to characterize a risk measure can be divided into three classes: rationality axioms, additivity axioms and technical axioms. First there are the basic rationality axioms such as monotonicity, which are satisfied by most risk measures. Additivity axioms deal with sums of risks. They describe the sensitivity of the risk measure with respect to risk aggregation. Let us mention the additivity for independent random variables, the additivity for comonotonic random variables, the subadditivity or the superadditivity. These additivity requirements often are the most characteristic for the corresponding risk measure. Finally, there are technical requirements, mostly continuity conditions. They are usually necessary for obtaining mathematical proofs and are typically difficult to validate or to explain economically.

Let us now detail some of the properties that risk measures may or may not satisfy:

Objectivity the risk measure $\varrho$ does not depend on the risk itself but only on its underlying distribution, i.e. $\varrho[X]=\varrho\left[F_{X}\right]$ where $F_{X}$ is the distribution function of $X$.

The objectivity requirement implies that risk measurement can be entirely based on distribution functions. This is usually the case in actuarial science. This condition ensures that $F_{X}$ contains all the information needed to measure the riskiness of $X$. This property is sometimes called "law invariance", and phrased as $X={ }_{d} Y \Rightarrow \varrho[X]=\varrho[Y]$ where $={ }_{d}$ denotes the equality in distribution.

Two monotonicity requirements can then be envisaged: either we impose that the risk measure $\varrho$ agrees with almost sure comparisons (weak monotonicity) or with stochastic dominance $\preceq_{\text {st }}$ (strong monotonicity).

Weak monotonicity for any $X$ and $Y, P[X \leq Y]=1$ implies $\varrho[X] \leq \varrho[Y]$.

Strong monotonicity for any $X$ and $Y$ such that $X \preceq_{\text {st }} Y$, i.e. $P[X>t] \leq P[Y>t]$ for all $t \in \mathbb{R}$, implies $\varrho[X] \leq \varrho[Y]$.

Comonotonicity is a useful notion in decision theory; see the review papers by DHAENE, Denuit, Goovaerts, KaAs \& Vyncke (2002a,b). Recall that the random variables $X$ and $Y$ are said to be comonotonic if they satisfy

$$
P[X \leq x, Y \leq y]=\min \{P[X \leq x], P[Y \leq y]\} \text { for all } x, y \in \mathbb{R}
$$


For two comonotonic random variables $X$ and $Y$, we have that $X \preceq_{\text {st }} Y \Leftrightarrow P[X \leq$ $Y]=1$, that is, $Y$ is greater than, or at least as large as, $X$ with probability one. In the absence of comonotonicity, weak monotonicity will not imply strong monotonocity. However, if $\varrho$ is objective, then both requirements have the same force. We refer the reader to BÄUERLE \& MüLLER (2005) for a careful examination of objectivity and monotonicity properties.

Positive homogeneity for any $a>0, \varrho[a X]=a \varrho[X]$.

In the literature it is often stated that positive homogeneity is equivalent with currency independency. This is however a wrong interpretation. Take as an example $\varrho[X]=$ $\mathbb{E}\left[(X-d)_{+}\right]$which is clearly currency independent (changing $X$ from dollars to euro only makes sense if also $d$ in changed in that way), but not positively homogenous. Hence, positive homogeneity is about multiplying all euro amounts of the claims by a constant factor. Let us also mention that future currencies are random so that the constant $a$ involved in the definition of positive homogeneity cannot be used to convert $X$ from euro to dollars, say. It is much more related to the "volume" of the risk and has a close relation with additivity for comonotonic risks (see below).

Translation equivariance for any $b \in \mathbb{R}, \varrho[X+b]=\varrho[X]+b$.

Translation equivariance might be considered desirable for a risk measure $\varrho$ used to calculate provisions or premiums. Indeed it seems to be reasonable when the risk contains a certain amount $b$ that $\varrho[b+X]=b+\varrho[X]$ in both cases. This is of course not the case for risk measures used for the calculation of solvency, regulatory or economic capital. These quantities constitute amounts of money for safety, in addition to the provisions and premiums. Such risk measures are of the form $\widetilde{\varrho}[X]=\varrho^{\prime}\left[(X-\varrho[X])_{+}\right]$for some appropriate risk measures $\varrho^{\prime}$ and $\varrho$. Consequently, if $\varrho$ is translation equivariant then $\widetilde{\varrho}$ satisfies $\widetilde{\varrho}[X+b]=\widetilde{\varrho}[X]$, so that we have translation invariance in this case. It is of course not forbidden to take translation equivariance as an axiom for the construction of risk measures in this situation, but it cannot be interpreted in the economic concept of solvency. The prescribed rules in the framework of Solvency 2 for the solvency capital (the difference between the Value-at-Risk at level 99.5\% and the Value-at-Risk at level $75 \%$ ) recognises this reality. Related of course is the requirement $\varrho[b]=b$ which can be defended in the case of insurance pricing (to avoid free lunches and/or the no-ripoff condition) but in the definition of a risk measure $\varrho[b]$ might be equal to a function $u(b)$, the utility. The coherence axioms seem to come closer to premium calculation principles than to risk measures useful in risk management, without being universally acceptable in this framework. As it will be explained further they relate more to uncertainty measures.

Combining positive homogeneity with translation equivariance guarantees that $\varrho$ is linear, that is, whatever $a>0, \varrho[a X+b]=a \varrho[X]+b$.

Subadditivity $\varrho[X+Y] \leq \varrho[X]+\varrho[Y]$ whatever the dependency structure of $X$ and $Y$. 
The rationale behind subadditivity can be summarized as "a merger does not create extra risk". Subadditivity reflects the idea that risk can be reduced by diversification. When equality holds, we speak of additivity. In this case, the dependence structure between $X$ and $Y$ is often specified: additivity for independent risks or additivity for comonotonic risks (see below). We refer the interested reader to DHAENE, LAEvEn, VAnduffel, Darkiewicz \& Goovaerts (2004) for a detailed discussion on the desirability of the subadditivity axiom.

In Artzner, Delbaen, Eber \& Heath (1999), a risk measure that satisfies the properties of monotonicity, positive homogeneity, translation equivariance and subadditivity is called a "coherent" risk measure. As explained below, coherent risk measures turn out to coincide with upper expectations introduced by HuBER (1981) in robust statistics. Coherent risk measures satisfying the objectivity requirement have been studied by KusuokA (2001); see also BÄUERLE \& Müller (2005) for an extension to unbounded random variables. The terminology "coherent" is misleading in the sense that it may suggest that any risk measure that is not "coherent" is always inadequate. It is worth to mention that coherency is defined with respect to a set of axioms, and no set is universally accepted. Modifying the set of axioms regarded as desirable leads to other risk measures that may be called coherent with just as much reason.

Convexity for any $\lambda \in[0,1], \varrho[\lambda X+(1-\lambda) Y] \leq \lambda \varrho[X]+(1-\lambda) \varrho[Y]$ whatever the dependency structure of $X$ and $Y$.

FÖLLMER \& SCHIED (2002) introduced the concept of convex risk measures, which satisfy the properties of monotonicity, translation equivariance and convexity. It has been further developed by FritTelli \& Rosazza Gianin (2002) and KrätTschmeR (2005). The class of coherent risk measures can be characterized as the class of convex risk measures that satisfy the positive homogeneity property. As the class of convex risk measures is an extension of the class of coherent risk measures, it is sometimes called the class of weakly coherent risk measures. It is worth to mention that the work by DEPREz \& GERBER (1985) already contains many nice results on convex risk measures.

Additivity for comonotonic risks $\varrho[X+Y]=\varrho[X]+\varrho[Y]$ for comonotonic random variables $X$ and $Y$.

Subadditivity and positive homogeneity are subject to debate. Whether subadditivity describes reality is in debate because it neglects the notion of residual risk completely. FÖLLMER \& SCHIED (2002) observed that the positive homogeneity and subadditivity properties make risk measures insensitive to liquidity risk. According to RoOTzEN \& KLÜPPELBERG (1999), subadditivity is a convenient mathematical property that does not hold in reality. The behavior of a risk measure with respect to the aggregation of risks is manifested by the award of diversification discounts and the imposition of penalties. Comonotonic additivity examines how the risk of the aggregate position $\varrho[X+Y]$ relates to the risks of the individual positions $\varrho[X]$ and $\varrho[Y]$. Comonotonic 
risks are bets on the same event and neither of them is a hedge against the other. Because of the no-hedge condition, no reduction in the risk-load is awarded for a combined policy, resulting in comonotonic additivity.

Additivity for independent risks $\varrho[X+Y]=\varrho[X]+\varrho[Y]$ for independent random variables $X$ and $Y$.

Additivity for independent risks is a reasonable requirement for a premium calculation principle, since it ensures that the policyholder has no interest in splitting the risk asking for coverage to several insurers. It is also justified by premium computation from top to down; see, e.g., Borch (1962) or Bühlmann (1985). The most general representation of risk measures that are additive for independent random variables is due to Gerber \& Goovaerts (1981) and is known as the mixed Esscher principle. Despite the numerous appealing features of the Esscher principle, it does not satisfy the strong monotonicity requirement. Counterexamples have been provided by VAN HeerwaArden, KaAs \& Goovaerts (1989). This is why Goovaerts, KaAs, LAEVEN \& TANG (2004) provided a new axiomatic characterization of risk measures that are additive for independent random variables, involving an axiom that guarantees monotonicity. The obtained risk measure is a restricted version of the mixed Esscher principle that can be regarded as an ordinary mixture of exponential premiums.

\subsection{Risk measures and stochastic orders}

Stochastic orders are partial orderings on spaces of probability distributions; see, e.g., Denuit, Dhaene, Goovaerts \& KaAs (2005) for a general account. A prominent example of stochastic order relation is the stochastic dominance $\preceq_{\text {st }}$ of which the definition has been recalled above. As we have seen from the definition of strong monotonicity in terms of $\preceq_{\text {st }}$, stochastic orders are closely related to risk measures. On the one hand, it seems reasonable to require that risk measures agree with some appropriate stochastic orders. On the other hand, several well-known stochastic orders can be characterized by means of risk measures.

Recall that $X$ is said to be smaller than $Y$ in the convex order, written as $X \preceq_{\mathrm{cx}} Y$, if $\mathbb{E}[X]=\mathbb{E}[Y]$ and $\mathbb{E}\left[(X-t)_{+}\right] \leq \mathbb{E}\left[(Y-t)_{+}\right]$for all $t$. Equivalently, $X \preceq_{\mathrm{cx}} Y \Leftrightarrow$ $\mathbb{E}[\phi(X)] \leq \mathbb{E}[\phi(Y)]$ for all convex functions $\phi: \mathbb{R} \rightarrow \mathbb{R}$, provided the expectations exist. Roughly speaking, convex functions are functions that take on their (relatively) larger values over regions of the form $(-\infty, a) \cup(b, \infty)$ for $a<b$. Therefore, if $X \preceq_{\mathrm{cx}} Y$ holds then $Y$ is more likely to take on "extreme" values than $X$. That is, $Y$ is "more variable" than $X$.

A closely related order relation is the increasing convex order, denoted as $\preceq_{\text {icx }}$. We have that $X \preceq_{\text {icx }} Y$ if there exists a third random variable $Z$ such that $X \preceq_{\text {st }} Z \preceq_{\text {cx }} Y$. Intuitively speaking, the random variable $Y$ such that $X \preceq_{\text {icx }} Y$ is thus "larger" and "more variable" than $X$.

A risk measure $\varrho$ is then said to preserve $\preceq_{\text {icx }}$ if for any $X$ and $Y$, one has that $X \preceq_{\text {icx }} Y \Rightarrow \varrho[X] \leq \varrho[Y]$. It can be shown that any risk measure that preserves $\preceq_{\text {icx }}$ 
and that is additive for comonotonic risks is subadditive. The preservation of $\preceq_{\mathrm{cx}}$ by convex risk measures is studied in B ̈̈UERLE \& MüLLER (2005).

In some works, the risk measure has to comply with other stochastic orders, for instance the 3-convex order of Denuit, Lefìvre \& Shaked (1998) in HürLimann (2004), the balayage (that is, the distribution free analogue of the increasing concave order, also called dilation) in LEITNER (2004). LEITNER (2006) considered the increasing concave order in the subclass of coherent risk measures. See also BäUERLE \& MüLLER (2005) for a general approach.

\subsection{Risk measures and economic decision under uncertainty}

Goovaerts, KaAs, Dhaene \& TAng (2004) gave several examples where evidently the properties that risk measures should have are determined by the realities of the actuarial applications (namely, insurance-reinsurance, premium calculation, premium calculation from top to down, capital allocation, solvency margin and setting provisions). See also Dhaene, Vanduffel, Tang, Goovaerts, KaAs \& Vyncke (2004).

Instead of stating a set of axioms that risk measures should satisfy and then determining mathematically their corresponding functional representation, one could determine the functional form of the risk measure via economic indifference arguments. For instance, the equivalent utility premium principle defined by BüHLMANN (1970) is obtained by considering an insurer whose preferences are characterized by expected utility (EU, in short). The distortion premium principle proposed by DENNEBERG (1990) and WANG (1996) can be obtained via indifference arguments based on distortion utility (DU, in short). The distortion-exponential premium principle has been similarly derived by Tsanakas \& Desli (2003) and Heilpern (2003) using rankdependent expected utility (RDEU, in short) theory. See also LuAn (2001). The present paper follows Denuit, DHAEne \& VAN Wouwe (1999) where only EU and DU were considered (with a brief excursion to RDEU).

Utility theories are typically presented in an axiomatic form. The axioms are intended as reasonable consistency requirements: the concept of rationality is embodied in the axioms, with the possible addition of the postulate of risk aversion. The difference is that here, axioms impose rational behavior in decision under uncertainty. As it was the case for the sets of axioms defining risk measures, the preference foundations typically use intuitive axioms such as transitivity, which refer to directly meaningful empirical properties of preferences, and technical axioms such as continuity, which describe the structural richness of the model and serve to simplify the mathematical analysis.

FishBURN (1982) and YAARI (1987) considered the following four axioms on the preference relation $\preceq$ :

Objectivity if $X$ and $Y$ have the same distribution function then the decision-maker is indifferent between $X$ and $Y$, that is, $X \preceq Y$ and $Y \preceq X$ both hold. 
Weak order $\preceq$ is complete, transitive and reflexive.

Strong monotonicity if $X \preceq_{\text {st }} Y$ then $X \preceq Y$.

Continuity the set $\{Y \mid Y \succeq X\}$ is closed for every $X$, the closure being often meant with respect to the topology of weak convergence, associated with the $L^{1}$ norm.

Then, a fifth axiom is added that specifies the particular form of the indicator of preferences. Whereas there is a rather large consensus about the four axioms listed above, the last one continues to be subject to debate. The EU axiomatization hinges on the independence axiom, which requires preferences to be separable accross mutually exclusive events. Alternative theories proposed by QUIGGIN (1982), YAARI (1987) and SCHMEIDLER (1989) replaced this condition with the comonotonic independence axiom, an intuitive and appealing condition requiring that the usual independence axiom only holds when hedging effects are absent.

The development of alternative paradigms for decision under uncertainty has been motivated by two main principles: uncertainty aversion should be expressed by models with not necessarily additive probabilities, and attitudes towards wealth and attitudes towards uncertainty (or risk) should be kept separate from each other. In this paper, we will consider in addition to the classical EU and DU theories several alternative paradigms for decision under uncertainty: the Choquet expected utility (CEU, in short) theory proposed by SCHMEIDLER (1989), the maximin expected utility (MEU, in short) theory proposed by GiLboA \& SchmeidLeR (1989) and the rank-dependent expected utility (RDEU, in short) theory proposed by QUIGGIN (1982). The application of the actuarial equivalent utility pricing principle in these paradigms will give most classes of risk measures considered so far in the financial mathematics literature.

\subsection{Risk and uncertainty}

Decision-making under risk and decision-making under uncertainty are differentiated according to the condition that in the former case the probabilities of events are given while in the latter case they are not. With regard to decision-making under uncertainty, models have been introduced which associate some kind of probabilities to events.

In the financial mathematics literature, and the actuarial papers in the realm of financial mathematics, the probability distribution of a risk does not need to be fixed (a risk is just a random variable defined on a measurable space, and the probability measure can be left unspecified). The majority of actuarial papers uses the framework of probability theory and statistics, in which a random variable is defined on a particular and fixed underlying probability space, not on just a measurable space with many possible probability measures. The probability measure may be unknown, but it is fixed and unique. This does not prevent that many weight functions operating on events can be used to calculate the premiums. For instance, the actuarial practice in life insurance consists in replacing the distribution of the remaining lifetime by a "more dangerous one" in order to generate a safety loading. We will also see below that in 
economics terminology, for example, a coherent risk measure is not a measure of risk but a measure of uncertainty.

Uncertainty is often represented with the help of capacities. Specifically, we assume that the uncertainty a decision maker faces can be described by a non-empty set of states, denoted as $\Omega$. This set may be finite or infinite. Associated with $\Omega$ is the set of events, taken to be a sigma-algebra of subsets of $\Omega$, denoted by $\mathcal{B}$. Capacities are realvalued functions defined on $\mathcal{B}$ that generalize the notion of probability distributions. Formally, a capacity is a normalized monotone set function. More precisely, $C: \mathcal{B} \rightarrow$ $[0,1]$ is a capacity if $C[\emptyset]=0, C[\Omega]=1$ and for any $A, B \in \mathcal{B}, A \subseteq B \Rightarrow C[A] \leq C[B]$. Otherwise, the capacity has little structure. In particular, the sum of the capacities of two disjoint subsets may exceed 1. Or the sum of the capacities of two subsets may be strictly less than the capacity of the union of these sets. The capacity $C$ is called coherent if there exists a non-empty set $\mathcal{M}$ of probability measures $P: \mathcal{B} \rightarrow[0,1]$ such that

$$
C[A]=\sup _{P \in \mathcal{M}} P[A] \text { for all } A \in \mathcal{B} .
$$

As we will see, coherent capacities are closely related to coherent risk measures.

To each capacity $C$ we can associate a dual capacity. The dual capacity of $C$, denoted as $\bar{C}$, is defined by

$$
\bar{C}[A]=1-C[\Omega \backslash A] \text { for all } A \in \mathcal{B} .
$$

The dual capacity is useful when losses are considered instead of gains. Given a coherent capacity $C$, a natural and widely used measure of the degree of uncertainty (or imprecision, vagueness, ambiguity) associated with an event $A \in \mathcal{B}$ is given by the interval

$$
\bar{C}[A], C[A]]=\left[\inf _{P \in \mathcal{M}} P[A], \sup _{P \in \mathcal{M}} P[A]\right] .
$$

The interval (1.1) captures uncertainty in the sense of Knight (1921). If $\bar{C}[A]=C[A]$, the event $A$ does not involve any vagueness and all priors $P \in \mathcal{M}$ agree on $A$.

Decisions under risk are usually performed in the EU or RDEU paradigms. In situations of uncertainty (i.e., in situations where there does not exist a given objective probability distribution available to the decision-maker), these paradigms are replaced by the subjective utility theory and the Choquet expected utility (CEU, in short), respectively.

\subsection{Aversion to risk and uncertainty}

There are many notions of risk aversion. Chateauneuf \& Cohen (2000) distinguish five kinds of risk aversion: weak, monotone, left monotone, right monotone and strong. In this paper, we restrict ourselves to the following two standard concepts of risk aversion. The first one defines an individual to be risk averse if the sure gain $\mathbb{E}[X]$ is always preferred to the random prospect $X$ itself. An alternative definition of risk aversion requires a risk averse individual to behave according to the concave order, 
denoted as $\preceq_{\mathrm{cv}}$, and defined by $X \preceq_{\mathrm{cv}} Y \Leftrightarrow-Y \preceq_{\mathrm{cx}}-X$. Alternatively, $X \preceq_{\mathrm{cv}} Y \Leftrightarrow$ $\mathbb{E}[\phi(X)] \leq \mathbb{E}[\phi(Y)]$ for all concave functions $\phi: \mathbb{R} \rightarrow \mathbb{R}$, provided the expectations exist. This order extends the concept of mean preserving increase in risk.

We thus have a concept of weak risk aversion $(\mathbb{E}[X]$ is preferred over $X)$ and a concept of strong risk aversion $\left(X \preceq_{\mathrm{cv}} Y \Rightarrow X\right.$ is preferred over $Y$ ). It is interesting to note that strong risk aversion is defined with the help of a stochastic order relation. Relations like the dispersive order have been used to define alternative attitudes towards risk; see, e.g., Chateauneuf \& Cohen (2000) and Chateauneuf, Cohen \& Meilijson (2004).

Aversion to uncertainty is commonly attributed to non-additivity of the subjective probabilities for ambiguous events: non-expected utility models posit that the decisionmaker has non-probabilistic beliefs. Uncertainty aversion then means that the decisionmaker tends to overweight events leading to inferior payoffs by applying non-additive subjective probabilities derived from a Choquet capacity.

\subsection{Outline of the paper}

This paper aims to demonstrate that, as pointed out by GEMAN (1999), in the fundamental subject of financial risk analysis, some valuable lessons may be drawn from insurance. Actuaries have used indifference arguments based on economic theories for decision under uncertainty for decades, after BüHLMANN (1970) introduced the equivalent utility principle in the context of setting insurance premiums. Considering this simple and appealing approach to risk measurement, most risk measures can be obtained in a way that enlightens their intrinsic properties. Unfortunately, apart from some authors including Föllmer \& SCHIED (2004), the close connections between risk measurement and economic decision theory have been disregarded by financial mathematicians, who independently developed their own axiomatic theories. This pa-

per tries to reconcile the two approaches, and demonstrates that the deficiencies of the risk measures recently studied in the financial mathematics literature come from the fact that they ignore the utility concept. Simple alternatives that can be found in the actuarial literature remedy to these problems, by considering the simple exponential utility functions.

\section{Equivalent utility risk measures in EU theory}

\subsection{EU theory}

The classical expected utility (EU, in short) theory is identified by the independence axiom. It is assumed that every EU decision-maker possesses some utility function $u(\cdot)$ and that he acts in order to maximize the expected utility. The prospect $X$ is then evaluated by

$$
\mathbb{U}_{E U}[X \mid u]=\int u(X) d P=\mathbb{E}[u(X)]
$$


and $X$ is preferred over $Y$ if $\mathbb{U}_{E U}[X \mid u] \geq \mathbb{U}_{E U}[Y \mid u]$. A classical reference for $\mathrm{EU}$ is Von Neumann \& Morgenstern (1947). The subjective utility theory of Savage (1954) for decision under uncertainty differs from EU only by its interpretation of the underlying probability distribution $P$.

If the decision maker, with initial wealth $w$ and utility function $u(\cdot)$, has to choose between random losses $X$ and $Y$, then he compares $\mathbb{U}_{E U}[w-X \mid u]$ with $\mathbb{U}_{E U}[w-Y \mid u]$ and chooses the loss which gives rise to the highest expected utility.

\subsection{Risk Aversion}

The two definitions of risk aversion are equivalent under EU theory, where they both coincide with the concavity of the utility function: for an EU decision-maker, weak risk aversion implies automatically strong risk aversion.

Note that in the EU framework, the agent's attitude towards risk and the agent's attitude towards wealth are forever bonded together (since they are both derived from the characteristics of $u$ ): risk aversion and diminishing marginal utility of wealth are synonymous. Nevertheless, risk aversion expresses an attitude towards risk while decreasing marginal utility expresses an attitude towards wealth. Thus, in EU theory, the concept of risk aversion being encapsulated in the utility function is a property of attitudes to wealth rather than of attitudes to risk.

\subsection{Equivalent EU risk measures}

Consider a decision-maker with initial wealth $w$ and with a utility function $u($.$) . Facing$ a random loss $X$, he sets its price for coverage $\varrho[X]$ as the solution of the equation

$$
\mathbb{U}_{E U}[w+\varrho[X]-X \mid u]=\mathbb{U}_{E U}[w \mid u]=u(w) .
$$

Condition (2.1) expresses that $\varrho[X]$ is fair in terms of utility : the right-hand side of (2.1) represents the utility of not not covering $X$; the left-hand side of (2.1) represents the expected utility of the decision-maker assuming the random financial loss $X$. Therefore (2.1) means that, provided an amount of $\varrho[X]$ is obtained when $X$ is covered, the expected utility of wealth with $X$ is equal to the utility without $X$. Putting $w=0$ and normalizing the utility function $u$, we get the so-called equivalent utility principle: $\varrho[X]$ calculated according to this principle is the root of the equation

$$
\mathbb{U}_{E U}[\varrho[X]-X \mid u]=0
$$

which can be interpreted as an equality between the expected utility of the income $\varrho[X]-X$ and the utility of not accepting the risk.

If we assume that the moment generating function of $X$ exists and that the utility function of the insurance company is of the form

$$
u(x)=\frac{1-\exp (-c x)}{c}, \quad x \geq 0,
$$


for some positive constant $c$, then (2.2) admits an explicit solution and $\varrho[X]$ can be expressed as

$$
\varrho[X]=\frac{1}{c} \ln \mathbb{E}[\exp (c X)]
$$

Note that the exponential principle (2.4) also possesses an appealing interpretation in terms of ruin theory; see, e.g., KaAs, Goovaerts, Dhaene \& Denuit (2001) or Tsanakas \& Desli (2003).

\section{Equivalent utility risk measures in DU theory}

\subsection{DU theory}

Modifying the independence axiom, YAARI (1987) showed that there must exist a distortion function $f$ such that a prospect $X$ is valued at its "distorted expectation" $\mathbb{U}_{D U}[X \mid f]$ defined as

$$
\mathbb{U}_{D U}[X \mid f]=-\int_{x=-\infty}^{0}\left(1-f\left(\bar{F}_{X}(x)\right)\right) d x+\int_{x=0}^{+\infty} f\left(\bar{F}_{X}(x)\right) d x
$$

where the distortion function $f:[0,1] \rightarrow[0,1]$ is non-decreasing with $f(0)=0$ and $f(1)=1$. Instead of using the tail probabilities $\bar{F}_{X}(x)$, the decision-maker uses the distorted tail probabilities $f\left(\bar{F}_{X}(x)\right)$. Yaari's model has been further developed by Rö̈LL (1987), and an alternative axiomatization is proposed in GURIEV (2001). We also refer to CARLIER \& DANA (2003) for interesting representations of $\mathbb{U}_{D U}[X \mid f]$.

\subsection{Risk Aversion}

Contrary to the EU case, the two concepts of risk aversion are no more equivalent in DU. For the weak risk aversion, it suffices that $f(p) \leq p, p \in[0,1]$, as we immediately find that

$$
\mathbb{U}_{D U}[X \mid f] \leq \mathbb{E}[X]=\mathbb{U}_{D U}[\mathbb{E}[X] \mid f]
$$

For the strong risk aversion, the distortion function $f$ has to be convex.

\subsection{Equivalent DU risk measures}

Let us introduce the dual distortion function $\bar{f}$ for each distortion function $f$ :

$$
\bar{f}(x)=1-f(1-x), \quad x \in[0,1] .
$$

The dual distortion function is again a distortion function. It is clear that $\overline{\bar{f}} \equiv f$ and that $f$ convex $\Leftrightarrow \bar{f}$ concave. Furthermore, we have that $\mathbb{U}_{D U}[-X \mid f]=-\mathbb{U}_{D U}[X \mid \bar{f}]$.

The equivalent DU risks measures are obtained as the solution of the indifference equation

$$
\mathbb{U}_{D U}[0 \mid f]=\mathbb{U}_{D U}[\varrho[X]-X \mid f]
$$


Solving (3.4) gives

$$
\begin{aligned}
\varrho[X] & =\mathbb{U}_{D U}[X \mid \bar{f}] \\
& =-\int_{-\infty}^{0}\left(1-\bar{f}\left(\bar{F}_{X}(x)\right)\right) \mathrm{d} x+\int_{0}^{\infty} \bar{f}\left(\bar{F}_{X}(x)\right) \mathrm{d} x .
\end{aligned}
$$

One immediately finds that $\bar{f}\left(\bar{F}_{X}(x)\right)$ is a non-increasing function of $x$ with values in the interval $[0,1]$. The risk measure $(3.5)$ can be interpreted as a "distorted expectation" of $X$, evaluated with a "distorted probability measure" in the sense of a Choquet-integral, see Denneberg (1994). Risk measures (3.5) are often called distortion risk measures. In the actuarial literature, distortion risk measures have been introduced by Denneberg (1990) and WANG (1996). Axiomatic characterizations of insurance prices leading to distorted premiums have been proposed in GoOvAERTs \& Dhaene (1998) and Wang, Young \& Panjer (1997).

Many risk measures can be cast into the form (3.5). Let us give some prominent examples.

Example 3.1 (Value-at-Risk). For any $p$ in $(0,1)$, the $p$-quantile risk measure (or Value-at-Risk) for a random variable $X$ is defined by $F_{X}^{-1}(p)=\inf \left\{x \in \mathbb{R} \mid F_{X}(x) \geq p\right\}$, $p \in(0,1)$. It can be shown that $F_{X}^{-1}(p)$ corresponds to the distortion function

$$
\bar{f}(q)=\left\{\begin{array}{l}
1 \text { if } q>1-p, \quad \text { for } 0 \leq q \leq 1 . \\
0 \text { otherwise, }
\end{array}\right.
$$

Example 3.2 (Tail-VaR). The Tail-VaR at level $p$, denoted by $\operatorname{TVaR}_{p}[X]$, is defined by

$$
\operatorname{TVaR}_{p}[X]=\frac{1}{1-p} \int_{p}^{1} F_{X}^{-1}(p) \mathrm{d} q, \quad p \in(0,1) .
$$

It is the arithmetic average of the quantiles of $X$, from $p$ on, and corresponds to the distortion function

$$
\bar{f}(x)=\min \left(\frac{x}{1-p}, 1\right), \quad 0 \leq x \leq 1 .
$$

VaR's are the building blocks of the distortion measures (3.5). To see it, it is enough to replace $\bar{f}\left(\bar{F}_{X}(x)\right)$ by $\int_{0}^{\bar{F}_{X}(x)} \mathrm{d} \bar{f}(q)$ in (3.5) and to revert the order of the integrations. One then finds that any distortion risk measure can be written as

$$
\varrho[X]=\int_{0}^{1} F_{X}^{-1}(1-q) \mathrm{d} \bar{f}(q) .
$$

Note that when the distortion function $\bar{f}$ is differentiable, (3.8) can be rewritten as

$$
\varrho[X]=E\left[F_{X}^{-1}(1-U) \bar{f}^{\prime}(U)\right] .
$$

Formulas (3.8)-(3.9) show that the spectral risk measures of ACERBI (2002) are particular cases of distortion risk measures. It can be further shown that coherent distortion 
risk measures can be represented as mixtures of Tail-VaR's, as pointed out by INUI \& KiJima (2005); see also BAssett, Koenker \& Kordas (2004) for portfolio allocation with mixtures of Tail-VaR's. As pointed out by PFLUG (2002), not all coherent risk measures can be represented as convex combinations of Tail-VaR's.

Distortion risk measures (3.5) with $\bar{f}$ concave are subadditive. Despite its wide adoption in practice, the VaR therefore fails to be subadditive. DANIELSSON, JoRGensen, Samorodnitsky, Sarma \& DE VRIEs (2005) explored the potential for violation of $\mathrm{VaR}$ subadditivity (both theoretically and by simulation). Their main finding was that for most practical applications $\mathrm{VaR}$ is subadditive. More precisely, they demonstrated that $\mathrm{VaR}$ is subadditive for the relevant tails of all fat tailed distributions, provided the tails are not super fat (i.e., distributions with so fat tails that the first moment is not defined, such as the Cauchy law, with tail index strictly less than 1). InUi, KiJima \& Kitano (2005) demonstrated the considerable bias of the $\mathrm{VaR}$ when used for a portfolio with fat-tailed distributions.

Note that the class of distortion risk measures (3.5) with concave $\bar{f}$ is a subset of the class of coherent risk measures, as is shown by the following example, taken from Dhaene, Vanduffel, Tang, Goovaerts, KaAs \& Vyncke (2004).

Example 3.3 (Dutch risk measure). For any random variable $X$, consider the risk measure

$$
\varrho[X]=\mathbb{E}[X]+\mathbb{E}\left[(X-\mathbb{E}[X])_{+}\right] .
$$

This is a particular case of the "Dutch premium principle", defined for non-negative random variables by KAAS, van HEERWAARDEN \& GoOvAERTs (1994).

The Dutch risk measure (3.10) is in general not additive for comonotonic risks. Hence, the Dutch risk measure (3.10) is an example of a risk measure that is coherent, although it is not a distortion risk measure. The example also illustrates the fact that coherent risk measures are not necessarily additive for comonotonic risks.

\section{Equivalent utility risk measures in CEU theory}

\subsection{CEU theory}

A possible device to construct a capacity is to take a probability measure $P$ on $\mathcal{B}$ together with a distortion function $f$, and then define $C=f \circ P$. Such a capacity $C$ is then called a distorted probability measure. Conversely, for a given capacity $C$, one may wonder whether there exists a probability measure $P$ such that $C=f \circ P$ for some distortion function $f$. The answer is in general negative: even if $\Omega$ is finite, there exist capacities that cannot be obtained as non-decreasing transformations of probability measures. See, e.g., LAEVEN (2005) for a simple counterexample.

SCHMEIDLER (1989) modified the independence axiom into comonotonic independence. This gave rise to the Choquet expected utility (CEU, in short) theory. In CEU 
theory, the prospect $X$ is evaluated by

$$
\begin{aligned}
\mathbb{U}_{C E U}[X \mid u, C] & =\int u(X) d C \\
& =\int_{-\infty}^{0}(C[u(X)>t]-1) d t+\int_{0}^{+\infty} C[u(X)>t] d t
\end{aligned}
$$

where $u$ is a utility of wealth function, $C$ is a capacity and integration is in the sense of Choquet. The functional $\mathbb{U}_{C E U}$ representing the preference relation in the CEU model is thus the Choquet integral of $u(X)$ with respect to the capacity $C$. Note that the integrals on the right-hand side are Riemann integrals and they are well defined since $C[u(X)>t]$ is a monotone function in $t$. Moreover, with $C$ defined directly on events, there is no necessity for any intermediate evaluation of probabilities.

\subsection{Uncertainty aversion}

Risk refers to situations where the perceived likelihoods of events of interest can be represented by probability distributions, whereas uncertainty refers to situations where the information available to the decision-maker is too imprecise to be summarized by a probability measure. SCHMEIDLER's (1989) CEU model takes place in the framework of uncertainty defined by Anscombe \& Aumann (1963).

Due to the absence of an objective probability distribution, the definition of uncertainty aversion has to be based on the random variables themselves. SCHMEIDLER (1989) suggests the idea that a convex combination of non comonotone random variables smooths the outcomes and makes an uncertainty-averse decision-maker better off. Specifically, a decision-maker is uncertainty-averse if, for every pair of random variables $X$ and $Y$, if $X$ is preferred over $Y$ then $\alpha X+(1-\alpha) Y$ is preferred over $Y$ for any $\alpha \in[0,1]$. The corresponding preferences are thus convex. Chateauneuf, DANA \& TALLON (2000) established that the decision-maker is uncertainty-averse if, and only if, the capacity $C$ is convex, that is,

$$
C[A \cup B]+C[A \cap B] \geq C[A]+C[B] \text { for all } A, B \in \mathcal{B},
$$

and the utility function $u$ is concave. See also LEITNER (2005) for related results.

If the reverse inequality holds in (4.1) then the capacity is called concave. Note that if the dual capacity $\bar{C}$ is convex then $C$ is concave. Furthermore, if $C$ is convex then $\bar{C}$ is concave and satisfies $C[A] \leq \bar{C}[A]$ for any $A \in \mathcal{B}$. The decision weights used in the computation of the Choquet integral will overweight large outcomes if $C$ is concave and will overweight low outcomes if $C$ is convex. Additivity is a special case that characterizes uncertainty neutrality. Probability distributions are special cases of capacities which are both concave and convex. In the case of a distorted probability $C[A]=f(P[A]), A \in \mathcal{B}$, if $f$ is convex then $C$ is convex, and if $f$ is concave then $C$ is concave.

The core of the capacity $C$ is defined as

$$
\operatorname{core}(C)=\{\text { probability measures } P \mid P[A] \geq C[A] \text { for all } A \in \mathcal{B}\} \text {. }
$$


Since SCHMEIDLER (1986), it is well-known that when the capacity is convex, its core is non-empty and the Choquet integral is given by

$$
\int u(X) d C=\inf _{P \in \operatorname{core}(C)} \int u(X) d P .
$$

This relates the CEU model to the MEU one, presented in the next section.

There are thus two properties of special interest concerning the uncertainty aversion in CEU theory, namely the non-emptiness of the core and the convexity of the capacity. Chateauneuf \& TAllon (2002) considered the preference for diversification in CEU. The agent is said to exhibit preference for diversification if, for any random variables $X_{1}, \ldots, X_{n}$ that are all considered as equivalent by the agent (i.e., $\mathbb{U}_{C E U}\left[X_{1} \mid u, C\right]=$ $\ldots=\mathbb{U}_{C E U}\left[X_{n} \mid u, C\right]$ in CEU $), \sum_{i=1}^{n} \alpha_{i} X_{i}$ is preferred over $X_{1}$ for any $\alpha_{1} \geq 0, \ldots, \alpha_{n} \geq$ 0 with $\sum_{i=1}^{n} \alpha_{i}=1$. This concept is obviously related to the convexity for risk measure. They established that preferences for portfolio diversification is equivalent to the agent having a convex capacity and a concave utility function.

\subsection{Equivalent CEU risk measures}

Most often in the financial literature, $u$ is taken to be the identity function and $C$ is assumed to be convex. We then get for the preference indicator simply the Choquet integral of $X$ with respect to $\mathrm{C}$, that is,

$$
\mathbb{U}_{C E U}[X \mid \text { identity, } C]=\int X d C=\int_{-\infty}^{0}(C[X>t]-1) d t+\int_{0}^{+\infty} C[X>t] d t .
$$

This integral is positively homogeneous, monotone and translation equivariant.

The risk measure $\varrho$ is then deduced from the indifference equation

$$
0=\mathbb{U}_{C E U}[\varrho[X]-X \mid \text { identity }, C] \Rightarrow \varrho[X]=-\int-X d C .
$$

Note that, in general, $\int X d C \leq-\int-X d C$. Equality holds for all random variables if, and only if, $C$ is additive. This inequality can be used to explain the bid-ask spread observed in some financial markets, according to CHATEAUnEUF, KAST \& LAPIED (1996). The integrals $\int X d C$ and $-\int-X d C$ are sometimes called lower and upper Choquet integrals, respectively. More precisely,

$$
\int X d C=\inf _{P \in \operatorname{core}(C)} \int X d P \text { and }-\int-X d C=\sup _{P \in \operatorname{core}(C)} \int X d P
$$

so that the length of the interval $\left(\int X d C,-\int-X d C\right)$ is intimately connected with the size of $\operatorname{core}(C)$.

After Denneberg (1990), the risk measure $\int X d C$ has been considered in insurance, e.g., by Wang (1996), Wang, Young \& Panjer (1997) and De WaegeNAERE, KAST \& LAPIED (2003) under the name of Choquet pricing. An overview 
paper of the applicability of this type of risk measures for solvency purposes is DHAENE, Vanduffel, Tang, Goovaerts, KaAs \& Vyncke (2004). Chateauneuf, Kast \& LAPIED (1996), WANG $(2000,2002)$ and HAmadA \& Sherris (2003) used the same pricing mechanism for financial markets; see also PELSSER (2004). This risk measure is continuous, monotonic and comonotonic additive. It is subadditive if, and only if, $C$ is concave; it is superadditive for convex $C$.

Castagnoli, Maccheroni \& Marinacci (2004) noticed that the presence on the market of assets without bid-ask spreads turn Choquet prices into standard expectations. More precisely, if an insurance company sets the safety loading according to a probability distortion in agreement with $\preceq_{\text {icv }}$ then it assigns either strictly positive safety loading to all contracts or zero safety loading to all contracts. Despite its theoretical interest, this result should not prevent to use Choquet risk measures (since actuarial risk theory imposes strictly positive safety loading, to avoid almost certain ruin).

KADANE \& WASSERMAN (1996) considered $\Omega=[0,1], \mathcal{B}$ the Borel subsets of $\Omega$ and symmetric capacities (that is, capacities $C$ such that $\mu[A]=\mu[B] \Rightarrow C[A]=C[B]$, with $\mu$ the Lebesgue measure). They characterized coherent symmetric capacities (as those with doubly star-shaped distortion functions; see KADANE \& WASSERMAN (1996) for a definition). If the capacity is further assumed to be concave then Section 4 in KADANE \& WASSERMAN (1996) indicates that the distortion functions $f(p)=\min \{p / \alpha, 1\}$ correspond to the extreme points of the set of all distortion functions. This directly implies that spectral risk measures can be represented as mixtures of Tail-VaR.

Note however that MARINACCI (1999) proved that symmetric and coherent Choquet capacities turn out to be additive under a fairly mild condition. More specifically, the existence of a single non-trivial unambiguous event (in the sense that the interval (1.1) reduces to a single point) is enough to make them additive. This is a very strong property since excluding the existence of even a single non-trivial unambiguous event seems in general a very demanding assumption (recall that in the additive case, all events are unambiguous). This is obviously related to the result of CASTAGNOLI, MACCHERONI \& MARINACCI (2004) mentioned above.

\section{Equivalent utility risk measures in MEU theory}

\subsection{MEU theory}

GilboA \& SchmeidleR (1989) suggested the Maximin expected utility (MEU, in short) model, also called multiprior model. In this context, preference relations have a representation by the functional

$$
\mathbb{U}_{M E U}[X \mid u, \mathcal{M}]=\inf \left\{\int u(X) d P \mid P \in \mathcal{M}\right\}
$$

where $u$ is a utility function and $\mathcal{M}$ is a closed and convex set of additive probability measures. Thus, CEU coincides with MEU when the set $\mathcal{M}$ is the core of $C$, that 
is, $\mathbb{U}_{C E U}[X \mid u, C]=\mathbb{U}_{M E U}[X \mid u, \operatorname{core}(C)]$. Moreover, when an arbitrary (closed and convex) set $\mathcal{M}$ is given and one defines $C[A]=\inf \{P[A] \mid P \in \mathcal{M}\}, C$ is not necessarily convex. Furthermore, even if $C$ happens to be convex, $\mathcal{M}$ does not have to be its core.

In addition to the usual assumptions on the preference relation as transitivity, completeness, continuity and weak monotonicity, GILBOA \& SchmeIDlen (1989) postulated uncertainty aversion and certainty-independence to derive MEU theory. The certainty-independence axiom is weaker than standard independence (since only mixings with degenerate distribution functions corresponding to certainty are considered).

\subsection{Equivalent MEU risk measures}

Taking $u(x)=x$ gives the class of coherent risk measures in the sense of ARTZnER, Delbaen, Eber \& Heath (1999). More precisely, the equivalent utility principle then yields

$$
0=\mathbb{U}_{M E U}[\varrho[X]-X \mid u, \mathcal{M}]=\inf _{P \in \mathcal{M}} \int(\varrho[X]-X) d P
$$

whence it follows that

$$
\varrho[X]=\sup _{P \in \mathcal{M}} \int X d P .
$$

This kind of risk measure has been used by CASTAGnoli, MaCcheroni \& MARINACCI (2002) to compute insurance premiums. More precisely, these authors established that if the insurance price system is internal (that is, the actuarial no-ripoff condition $\varrho[X] \leq \max [X]$ is satisfied), sublinear (that is, positively homogeneous and subadditive), consistent with the market, and satisfying two continuity axioms then insurance prices are the maximum of their expected payments with respect to a family of risk neutral probabilities.

Huber (1981) already considered functionals (5.1) in robust statistics. The elements of $\mathcal{M}$ are called generalized scenarios in Artzner, Delbaen, Eber \& Heath (1999). Huber (1981) proved for the case of a finite set $\Omega$, that a risk measure is coherent if, and only if, it has an upper expectation representation. This result remains valid for more general spaces (see Delbaen (2002)), though in that case "the important conceptual aspects are buried under a mass of technical complications of a measure theoretic and topological nature," as is stated by HuBER (1981).

Risk measures of the form (5.1) have been considered for a long time in actuarial science, especially when $\mathcal{M}$ is a moment space (or more generally, when $\mathcal{M}$ is defined by a set of integral constraints). See, e.g., Section 5.3 of Goovaerts, De Vijlder \& HAEZENDONCK (1984) for a detailed account. 


\section{Equivalent utility risk measures in RDEU theory}

\subsection{RDEU theory}

In this section, we present a theory which combines the EU and the DU assumptions, to a certain extent. The rank-dependent expected utility (RDEU, in short) theory was first proposed by QUIGGIN (1982). It is a generalization of EU and DU theories that preserves the properties of continuity, transitivity and weak monotonicity. We do not give the set of axioms connected with RDEU since the modified version of the independence axiom appearing in QUIGGIN (1982) does not admit a clear behavioral interpretation. For other sets of axioms, see e.g. Chateauneuf \& Wakker (1999) and ABDellaoui (2002). The RDEU approach combines the utility function $u(\cdot)$ from EU with a distortion function $f(\cdot)$ from DU. For interesting applications to insurance, see, e.g., Chateauneuf, Cohen \& Meilijson (1997).

Under the rank-dependent expected utility model, a decision-maker is characterized by a utility function $u$ (that plays the role of utility on certainty) in conjunction with a distorted function $f$ (that plays the role of a probability perception function). Such a decision-maker prefers the fortune $Y$ to the fortune $X$ if, and only if, $\mathbb{U}_{R D E U}[X \mid u, f] \leq$ $\mathbb{U}_{R D E U}[Y \mid u, f]$ where

$$
\mathbb{U}_{R D E U}[X \mid u, f]=\int_{t=-\infty}^{0}(f(P[u(X)>t])-1) d t+\int_{t=0}^{+\infty} f(P[u(X)>t]) d t
$$

with a similar expression for $Y$. It is easy to see that if $f(p)=p$, we get the EU model, that is, $\mathbb{U}_{R D E U}[X \mid u$, identity $]=\mathbb{U}_{E U}[X \mid u]$, whereas if $u(x)=x$ we get the DU paradigm, that is, $\mathbb{U}_{R D E U}[X \mid$ identity, $f]=\mathbb{U}_{D U}[X \mid f]$.

Just as in EU, the utility function $u$ is normally taken to be concave or linear. There is less consensus concerning the appropriate shape for the distortion function $f$. QUIGGIN (1982) originally proposed an S-shaped function, overweighting extreme low probability outcomes. Other authors suggested to consider convex distortion functions $f$ (this property is akin to risk aversion, as shown above).

Simple distortion functions are those given by the one-parameter family $f_{\alpha}(p)=$ $\min \{p / \alpha, 1\}$ for some $\alpha \in[0,1]$. In this case, we have

$$
\mathbb{U}_{R D E U}\left[X \mid u, f_{\alpha}\right]=\frac{1}{\alpha} \int_{0}^{\alpha} u\left(F_{X}^{-1}(p)\right) d p
$$

This family has been considered, e.g., in Bassett, Koenker \& Kordas (2004).

Remark 6.1. Following the usage of KNIGHT (1921) in distinguishing uncertainty (i.e., ambiguity) and risk, GilboA (1987) and SchmeIDLER (1989) do not take objective probabilities as given but consider subjective probabilities. Capacities are then used and prospects are compared on the basis of Choquet integrals. WAKKER (1990) established that, provided weak monotonicity is fulfilled, this approach is equivalent to RDEU. The subtle difference between the two approaches can be summarized as follows: in RDEU, decision-makers know the objective probabilities but distort them 
to choose among risky prospects. In CEU, decision-makers use their own subjective probabilities, derived from some capacity.

\subsection{Risk aversion}

As RDEU distinguishes attitudes to outcomes and attitudes to probabilities, risk aversion in RDEU must combine two different concepts. First, there is outcome risk aversion, associated with the idea that the marginal utility of wealth is declining. This is the standard notion of risk aversion from the EU theory defined by concavity of the utility function. Second, there are attitudes specific to probability preferences. Risk aversion in probability weighting corresponds to pessimism: the decision-maker adopts a set of decision weights that yields an expected value for a transformed risky prospect lower than the mathematical expectation. An alternative, more restrictive, characterization of pessimism leads to a definition of risk aversion in terms of $\preceq_{\text {icv }}$. Note that the restriction to concave utility functions does not prohibit risk seeking behaviors in RDEU. It is indeed possible to model risk seeking with diminishing marginal utility of wealth, an attractive feature of RDEU.

The transformation $f$ may be regarded as pessimistic if $f(p) \leq p$ for all $p$. This comes from the fact that $f(p) \leq p$ for all $p$ if, and only if, for any random variable $X$ and concave utility $u$

$$
\mathbb{U}_{R D E U}[X \mid u, f] \leq \mathbb{U}_{R D E U}[\mathbb{E}[X] \mid u, f]=u(\mathbb{E}[X]),
$$

that is, $\mathbb{E}[X]$ is preferred over $X$. Therefore, concavity of $u$ and pessimism are reasonable conditions for risk aversion.

Let us now turn to the definition of risk aversion as preservation of $\preceq_{\text {icv }}$. It can be shown that a decision-maker will be coherent with $\preceq_{\text {icv }}$ if, and only if, $u$ is concave and $f$ is convex. Convexity of the distortion function $f$ implies a strong form of pessimism that can be roughly summarized as follows: the worst outcome is overweighted most, and the best outcome underweighted most. Unlike the situation in EU theory, preservation of $\preceq_{\text {icv }}$ is not equivalent to preference for certainty over risk.

\subsection{Equivalent RDEU risk measures}

Heilpern (2003) and Tsanakas \& Desli (2003) introduce a class of risk measures which can be considered as the solutions of the indifference equations RDEU. Lemma 1 in HEILPERN (2003) indicates that provided $u$ is non-decreasing and concave, $\mathbb{U}_{R D E U}[X \mid u, f] \leq u\left(\mathbb{U}_{D U}[X \mid f]\right)$ whatever the distortion function $f$. The equivalent RDEU risk measure solves

$$
u(0)=\mathbb{U}_{R D E U}[\varrho[X]-X \mid u, f] .
$$

In general, there is no explicit solution to this equation. A notable exception is with utility function of the form (2.3) for which

$$
\varrho[X]=\frac{1}{c} \ln \left(\int_{0}^{+\infty} \bar{f}(P[\exp (c X)>t]) d t\right) .
$$


The equivalent utility risk measures solving (6.1) are much more subtle than those obtained before. Indeed, provided $u$ is non-decreasing and concave and $f$ is a convex distortion function, HEILPERN (2003, Theorem 1) proved that the resulting risk measure is translation equivariant, preserves $\preceq_{\text {icx }}$ and does not entail unjustified safety loading, that is $\varrho[c]=c$ for all real constants $c$. If $u(x)=x$, it is moreover positively homogeneous, comonotonic additive and subadditive. Note that in this case, we are back to the DU paradigm. It is additive for independent random variables only in very special cases (broadly speaking, if $u$ is linear or exponential and $f$ is the identity).

In particular, the risk measure (6.2) is additive for independent random variables only if $\bar{f}(p)=p$ (that is, in the EU case). Moreover, the risk measure (6.2) is strongly monotone, translation equivariant and convex. TsanakAs \& Desli (2003) studied in detail the properties of this risk measure regarding sensitivity to portfolio size and to risk aggregation. They conclude that this risk measure behaves approximately as a coherent risk measure for smaller portfolios of risks, while for larger portfolios the risk aversion induced by the utility function becomes prevalent, and the sensitivity to liquidity and risk aggregation issues gradually increases.

\section{Conclusion}

In this paper, we have derived the great majority of the classical risk measures using the actuarial equivalent utility principle in different theories for decision under risk and uncertainty. This makes clear the economic rationale behind these risk measures. This also suggests that the drawbacks of these risk measures come from the linearity of the associated utility function. The risk measure (6.2) proposed by TSANAKAS \& DESLI (2003) seems to be more appropriate than those found so far in the financial literature because it involves a nonlinear utility function.

\section{Acknowledgements}

The financial support of the Belgian Government under the contract "Projet d'Actions de Recherche Concertées" ARC 04/09-320 is gratefully acknowledged by Michel Denuit, who also thanks the Banque Nationale de Belgique for financial support under grant "Risk Measures and Economic Capital".

\section{References}

[1] Abdellaoui, M. (2002). A genuine rank-dependent generalization of the von Neumann-Morgenstern expected utility theorem. Econometrica 70, 717-736.

[2] Acerbi, C. (2002). Spectral measures of risk: A coherent representation of subjective risk aversion. Journal of Banking and Finance 26, 1505-1518. 
[3] Anscombe, F.J., \& Aumann, R.J. (1963). A definition of subjective probability. Annals of Mathematical Statistics 34, 199-205.

[4] Artzner, Ph., Delbaen, F., Eber, J.-M., \& Heath, D. (1999). Coherent risk measures. Mathematical Finance 9, 203-228.

[5] Bassett, G.W., Koenker, R., \& Kordas, G. (2004). Pessimistic portfolio allocation and Choquet expected utility. Journal of Financial Econometrics 2, 477-492.

[6] BäUerle, N., \& Müller, A. (2005). Stochastic orders and risk measures: Consistency and bounds. Insurance: Mathematics \& Economics, in press.

[7] Borch, K. (1962). Equilibrium in a reinsurance market. Econometrica 3, 424-444.

[8] Bühlmann, H. (1970). Mathematical Methods in Risk Theory. Springer Verlag, New York.

[9] Bühlmann, H. (1985). Premium calculation from top down. Astin Bulletin 15, 89-101.

[10] Carlier, G., \& Dana, R.A. (2003). The core of convex distortion of a probability. Journal of Economic Theory 113, 199-222.

[11] Castagnoli, E., Maccheroni, F., \& Marinacci, M. (2002). Insurance premia consistent with the market. Insurance: Mathematics and Economics 31, 267284 .

[12] Castagnoli, E., Maccheroni, F., \& Marinacci, M. (2004). Choquet insurance pricing: A caveat. Mathematical Finance 14, 481-485.

[13] Chateauneuf, A., \& Cohen, M. (2000). Choquet expected utility model: A new approach to individual behavior under uncertainty and to social welfare. In Grabish, M., Mirofushi, T., Sugeno, M. editors, Fuzzy Measures and Integrals, Kluwer Academic Publishers pp. 289-313.

[14] Chateauneuf, A., Cohen, M., \& Meilijson, I. (1997). New tools to better model behavior under risk and uncertainty: An overview. Finance 18, 25-46.

[15] Chateauneuf, A., Cohen, M., \& Meilijson, I. (2004). Four notions of meanpreserving increases in risk, risk attitudes and applications to the rank-dependent expected utility model. Journal of Mathematical Economics 40, 547-571.

[16] Chateauneuf, A., Dana, R.A., \& Tallon, J.-M. (2000). Optimal risk sharing rules and equilibria with Choquet-expected-utility. Journal of Mathematical Economics 34, 191-214. 
[17] Chateauneuf, A., Kast, R., \& Lapied, A. (1996). Choquet pricing for financial markets with frictions. Mathematical Finance 6, 323-330.

[18] Chateauneuf, A., \& Tallon, J.-M. (2002). Diversification, convex preferences and non-empty core in the Choquet expected utility model. Economic Theory 19, 509-523.

[19] Chateauneuf, A., \& Wakker, P.P. (1999). An axiomatization of cumulative prospect theory for decision under risk. Journal of Risk and Uncertainty 18, 137145 .

[20] Danielsson, J., Jorgensen, B.N., Samorodnitsky, G., Sarma, M., \& DE VRIES, C.G. (2005). Subadditivity re-examined: The case for Value-at-Risk. Manuscript.

[21] Delbaen, F. (2002). Coherent risk measures on general probability spaces. In Essays in Honour of Dieter Sondermann, Springer Verlag.

[22] Denneberg, D. (1990). Distorted probabilities and insurance premiums. Methods of Operations Research 63, 3-5.

[23] Denneberg, D. (1994). Non-Additive Measure and Integral. Kluwer Academic Publishers, Boston.

[24] Denuit, M., Dhaene, J., Goovaerts, M.J., \& KaAs, R. (2005). Actuarial Theory for Dependent Risk: Measures, Orders and Models. Wiley, New York.

[25] Denuit, M., Dhaene, J., \& Van Wouwe, M. (1999). The economics of insurance: A review and some recent developments. Bulletin of the Swiss Association of Actuaries, 137-175.

[26] Denuit, M., Lefìvre, Cl., \& Shaked, M. (1998). The s-convex orders among real random variables, with applications. Mathematical Inequalities and Their Applications 1, 585-613.

[27] Deprez, O., \& Gerber, H.U. (1985). On convex principles of premium calculation. Insurance: Mathematics \& Economics 4, 179-189.

[28] De Waegenaere, A., Kast, R., \& Lapied, A. (2003). Choquet pricing and equilibrium. Insurance: Mathematics and Economics 32, 359-370.

[29] Dhaene, J., Denuit, M., Goovaerts, M.J., KaAs, R., \& Vyncke, D. (2002a). The concept of comonotonicity in actuarial science and finance: Theory. Insurance: Mathematics and Economics 31, 3-33.

[30] Dhaene, J., Denuit, M., Goovaerts, M.J., KaAs, R., \& Vyncke, D. (2002b). The concept of comonotonicity in actuarial science and finance: Applications. Insurance: Mathematics and Economics 31, 133-161. 
[31] Dhaene, J., Laeven, R.J.A., Vanduffel, S., Darkiewicz, G. \& GoovaerTs, M.J. (2004) Can a coherent risk measure be too subadditive? Research Report OR 0431, Department of Applied Economics, K.U.Leuven.

[32] Dhaene, J., Vanduffel, S., Tang, Q., Goovaerts, M.J., Kaas, R., \& Vyncke, D. (2004). Capital requirements, risk measures and comonotonicity. Belgian Actuarial Bulletin 4, 53-61

[33] Fishburn, P.C. (1982). The Foundations of Expected Utility. Reidel Publishing Co, Dordrecht.

[34] Föllmer, H., \& Schied, A. (2002). Convex measures of risk and trading constraints. Finance and Stochastics 6, 429-447.

[35] Föllmer, H., \& Schied, A. (2004). Stochastic Finance (2nd Edition). De Gruyter, Berlin.

[36] Frittelli, M., Rosazza Gianin, E. (2002). Putting order in risk measures. Journal of Banking \& Finance 26, 1473-1486.

[37] Geman, H. (1999). Learning about risk: Some lessons from insurance. European Finance Review 2, 113-124.

[38] Gerber, H.U., \& Goovaerts, M.J. (1981). On the representation of additive principles of premium calculation. Scandinavian Actuarial Journal, 221-227.

[39] GilboA, I. (1987), Expected utility with purely subjective non-additive probabilities. Journal of Mathematical Economics 16, 65-88.

[40] Gilboa, I., \& Schmeidler, D. (1989). Maxmin expected utility with a nonunique prior. Journal of Mathematical Economics 18, 141-153.

[41] Goovaerts, M.J., De Vijlder, F., \& Haezendonck, J. (1984). Insurance Premiums. North-Holland, Amsterdam.

[42] Goovaerts, M.J., \& Dhaene, J. (1998). On the characterization of Wang's class of premium principles. Transactions of the 26th International Congress of Actuaries 4, 121-134.

[43] Goovaerts, M.J., Kaas, R., Dhaene, J., \& Tang, Q. (2003). A unified approach to generate risk measures. ASTIN Bulletin 33, 173-191.

[44] Goovaerts, M.J., Kaas, R., Dhaene, J., \& Tang, Q. (2004). Some new classes of consistent risk measures. Insurance: Mathematics and Economics 34, 505-516.

[45] Goovaerts, M.J., Kaas, R., Laeven, R.J.A., \& Tang, Q. (2004). A comonotonic image of independence for additive risk measures. Insurance: Mathematics and Economics 35, 581-594. 
[46] Guriev, S. (2001). On microfoundation of the dual theory of choice. The Geneva Papers on Risk and Insurance Theory 26, 117-137.

[47] Hamada, M., \& Sherris, M. (2003). Contingent claim pricing using probability distortion operators: Methods from insurance risk pricing and their relationship to financial theory. Applied Mathematical Finance 10, 19-47.

[48] Heilpern, S. (2003). A rank-dependent generalization of zero-utility principle. Insurance: Mathematics and Economics 33, 67-73.

[49] Huber, P.J. (1981). Robust Statistics. Wiley, New York.

[50] Hürlimann, W. (2004). Distortion risk measures and economic capital. North American Actuarial Journal 8, 86-95.

[51] Inui, K., \& KiJima, M. (2005). On the significance of expected shortfall as a coherent risk measure. Journal of Banking and Finance 29, 853-864.

[52] Inui, K., Kijima, M., \& Kitano, A. (2005). VaR is subject to a significant positive bias. Statistics and Probability Letters 72, 299-311.

[53] Kaas, R., Goovaerts, M.J., Dhaene, J., \& Denuit, M. (2001). Modern Actuarial Risk Theory. Kluwer Academic Publishers, Dordrecht.

[54] Kaas, R., Van Heerwaarden, A.E., \& Goovaerts, M.J. (1994). Ordering of Actuarial Risks. CAIRE Educations Series, Brussels.

[55] Kadane, J., \& Wasserman, L.A. (1996). Symmetric, coherent, Choquet capacities. Annals of Statistics 24, 1250-1264.

[56] Knight, F.H. (1921). Risk, Uncertainty, and Profit. Houghton Mifflin Company, Boston.

[57] Kusuoka, S. (2001). On law invariant coherent risk measures. Advances in Mathematical Economics 3, 83-95.

[58] KRÄTschmeR, V. (2005). Robust representation of convex risk measures by probability measures. Finance and Stochastics 9, 597-608.

[59] Laeven, R.J.A. (2005). Essays on Risk Measures and Stochastic Dependence. Tinbergen Institute Research Series 360, Thela Thesis, Amsterdam.

[60] Leitner, J. (2004). Balayage monotonous risk measures. International Journal of Theoretical and Applied Finance 7, 887-900.

[61] Leitner, J. (2005). Dilation monotonous Choquet integrals. Journal of Mathematical Economics 41, 994-1006. 
[62] Leitner, J. (2006). A short note on second order stochastic dominance preserving coherent risk measure. Mathematical Finance, in press.

[63] LuAn, C. (2001). Insurance premium calculations with anticipated utility theory. ASTIN Bulletin 31, 23-35.

[64] Marinacci, M. (1999). Upper probability and additivity. Sankhya - Series A 61, 358-361.

[65] Pelsser, A.A.J. (2004). On the applicability of the Wang transform for pricing financial risks. Working Paper, Erasmus University Rotterdam.

[66] Pflug, G.C. (2002). Coherent risk measures and convex combinations of the conditional Value at Risk. Austrian Journal of Statistics 31, 73-75.

[67] Quiggin, J. (1982). A theory of anticipated utility. Journal of Economic Behavior and Organisation 3, 323-343.

[68] Rö̈Ll, A. (1987). Risk aversion in Quiggin and Yaari's rank-order model of choice under uncertainty. Economic Journal 97, 143-159.

[69] Rootzen, H., \& Klüppelberg, C. (1999). A single number cannot hedge against economic catastrophes. Ambio-Royal Swedish Academy of Science 8, 550555.

[70] Savage, L. (1954). The Foundations of Statistics. Wiley, New York.

[71] Schmeidler, D. (1986). Integral representation without additivity. Proceedings of the American Mathematical Society 97, 253-261.

[72] Schmeidler, D. (1989). Subjective probability and expected utility without additivity. Econometrica 57, 571-587.

[73] Tsanakas, A., \& Desli, E. (2003). Risk measures and theories of choice. British Actuarial Journal 9, 959-981.

[74] Van Heerwaarden, A.E., KaAs, R., \& Goovaerts, M.J. (1989). Properties of the Esscher premium calculation principle. Insurance: Mathematics \& Economics 8, 261-267.

[75] Von Neumann, J., \& Morgenstern, O. (1947). Theory of Games and Economic Behavior (2nd edition). Princeton University Press, Princeton.

[76] WAKker, P.P. (1990). Under stochastic dominance Choquet-expected utility theory and anticipated utility are identical. Theory and Decision 29, 453-463.

[77] WAKker, P.P. (2004). Preference axiomatizations for decision under uncertainty. In Gilboa, I. editor, Uncertainty in Economic Theory: A Collection of Essays in Honor of David Schmeidler's 65th Birthday, Routledge, pp. 20-35. 
[78] Wang, S.S. (1996). Premium calculation by transforming the layer premium density. ASTIN Bulletin 26, 71-92.

[79] Wang, S.S. (2000). A class of distortion operators for pricing financial and insurance risks. Journal of Risk and Insurance 67, 15-36.

[80] Wang, S.S. (2002). A universal framework for pricing financial and insurance risks. ASTIN Bulletin 32, 213-234.

[81] Wang, S.S., Young, V.R., \& Panjer, H.H. (1997). Axiomatic characterization of insurance prices. Insurance : Mathematics \& Economics 21, 173-183.

[82] YAARI, M.E. (1987). The dual theory of choice under risk. Econometrica 55, 95-115. 